\title{
THE NATURAL SETS OF WANG'S PREMIUM PRINCIPLE ${ }^{1}$
}

\author{
BY \\ XIAN-YI WU \\ East China Normal University, Shanghai, China
}

\begin{abstract}
Recently, Wang's premium principle (Wang, 1995, 1996) has been discussed by many authors. Considerable attention has been given to the conditions under which Wang's premium principle can be reduced to the standard deviation premium principle. In this paper, we have got two results on this problem. One is that the natural set is a location-scale family if Wang's premium principle can be reduced to the SD premium principle for all surjective distortions. The other is that the natural set is a location-scale family for all power distortions.
\end{abstract}

\section{KEYWORDS AND PHRASES}

Premium principle, distortion function, proportional hazard transformation, natural set, location-scale family, Laplace transformation.

\section{INTRODUCTION}

Recently, Wang's premium principle (Wang, 1995, 1996) has been discussed by many authors, e.g., Wang, Young and Panjer (1997) and Wang and Young (1998). Christofides (1998) found that for some distribution families, the proportional hazards $(\mathrm{PH})$ premium principle, as a special case of Wang's premium principle when the distortion is a power function, can be reduced to the well known standard deviation (SD) premium principle. He conjectures that for a parametric distribution family with constant skewness, the $\mathrm{PH}$ premium principle reduces to the SD principle. Young (1999) showed that Christofides' conjecture is true for location-scale families and for certain other families, but false in general. Wang (2000) introduced the concept of natural set for the distortion, and showed that for a fixed distortion, the natural set on which Wang's premium principle reduces to the SD premium principle is a union of location-scale families which satisfies some condition. Furthermore, he showed

\footnotetext{
1 Project 19831020 Supported by National Natural Science Foundation of China.
} 
that the natural set is a location-scale family if Wang's premium principle reduces to the $\mathrm{SD}$ premium principle for all distortions, or especially for all two-step-up distortions in which a two-step-up distortion is a distortion that takes only the values 0 and 1 . In fact, his results mean that corresponding to each distortion there is a natural set (a union of location-scale families), and the intersection of all these natural sets for all two-set-up distortions is a locationscale family. He proposed a question of whether the natural set is a locationscale family for all power distortions. In this paper, we will provide further discussion on this topic. We have the following two results. One is that the natural set is a location-scale family if Wang's premium principle can be reduced to the SD premium principle for all surjective distortions. Note that any twostep-up distortion is not surjective. The other is that the natural set is a locationscale family for all power distortions.

\section{Results}

As we all know, the standard deviation premium principle is that

$$
\pi(X)=E(X)+\lambda \sqrt{\operatorname{Var}(X)}
$$

for some $\lambda>0$, where $\pi(X)$ is the premium charged for insurance risk $X$. Wang's $(1995,1996)$ premium principle gives the premium

$$
H_{g}(X)=\int_{-\infty}^{0}\left\{g\left[S_{X}(t)\right]-1\right\} d t+\int_{0}^{\infty} g\left[S_{X}(t)\right] d t
$$

where $S_{X}(t)=P(X>t)$ is the decumulative distribution function (d.d.f., or survival function) of $X$, and the distortion $g$ is a non-decreasing function from $[0,1]$ onto itself. If $g$ is a power function $g(w)=w^{c}$, then $g\left[S_{X}(t)\right]$ is referred as a proportional hazard transformation of $S_{X}(t)$ (Wang, 1995, 1996), and $H_{g}(X)$ is called proportional hazard $(\mathrm{PH})$ premium of $X$. For convenience, denote the PH premium of a random variable $X$ as $H_{c}(X)$ for any fixed $c$.

Let $\mathrm{g}$ be a fixed distortion, then it is obvious that Wang's premium principle can reduce to the $\mathrm{SD}$ principle in a set, $F$, of distributions, if and only if, for any $X \in F$ and $Y \in F$, the following equation holds,

$$
\frac{H_{g}(X)-E(X)}{\sqrt{\operatorname{Var}(X)}}=\frac{H_{g}(Y)-E(Y)}{\sqrt{\operatorname{Var}(Y)}}
$$

Now we give the definition of natural set of a distortion function which is introduced by Wang (2000). 
Definition 1. Let $\Omega$ be the set of all distributions. The natural set of a distortion $g$ with respect to distribution $X \in \Omega$, denoted as $N_{g}(X)$, is defined as

$$
N_{g}(X)=\left\{Y: \frac{H_{g}(X)-E(X)}{\sqrt{\operatorname{Var}(X)}}=\frac{H_{g}(Y)-E(Y)}{\sqrt{\operatorname{Var}(Y)}}, Y \in \Omega\right\}
$$

If

$$
\lambda=\lambda(X)=\frac{H_{g}(X)-E(X)}{\sqrt{\operatorname{Var}(X)}}
$$

we also write $N_{g}(X)$ as $N_{g}(\lambda)$.

Obviously, a fixed g may have many natural sets with each set corresponding to different parameter $\lambda$ 's. The proposition 1 in Wang (2000) said that $N_{g}(X)$ is a union of some location-scale families.

Definition 2. $g$ is called a surjective distortion if $g$ is a non-decreasing surjection from $[0,1]$ onto itself. We denote the set of all distortion function as $G$, the set of all two-step-up distortions as $T$, the set of all surjective distortions as $S$ and the set of all proportional hazard transformations (power distortions) as $P$.

Obviously, $P, T$ and $S$ are all subsets of $G$ and a distortion is a surjective distortion if and only if $g$ is a continuous distortion satisfying $g(0)=0$ and $g(1)=1$.

By using a two-step-up distortion, Wang (2000) showed that the natural set on which Wang's premium principle can reduce to the standard premium principle for all distortions (or for all two-step-up distortions) is a location-scale family. It means that both $\bigcap_{g \in G} N_{g}(X)$ and $\bigcap_{g \in T} N_{g}(X)$ are location-scale families. In fact, the subscript for which the intersection is carried on need not go through the set $G$ of all distortions or the set $T$ of all two-step-up distortions. We will prove that $\bigcap_{g \in S} N_{g}(X)$ is a location-scale family, and if $X$ has continuous d.d.f. and convex support set, then $\bigcap_{g \in P} N_{g}(X)$ is also a location-scale family. Before the main results, we present the following two lemmas.

Lemma 1. Let $S(x)$ be a decumulative distribution function of a random variable $X$, and

$$
g(w)= \begin{cases}0, & 0 \leq w \leq A \\ 1, & A<w \leq 1\end{cases}
$$

be a two-step-up distortion function for $A \in(0,1)$. Let $u=\inf \{t: S(t) \leq A\}$, then Wang's premium $H_{g}(X)=u$. 
Proof: Since the decumulative distribution function is right continuous, $S(t)$ is larger than $A$ when $t<u$ and smaller than or equal to $A$ when $t \geq u$. Therefore we have

$$
g[S(t)]= \begin{cases}1, & t<u \\ 0, & t \geq u\end{cases}
$$

If $u>0$, we have

$$
H_{g}(X)=\int_{-\infty}^{0}\left\{g\left[S_{X}(t)\right]-1\right\} d t+\int_{0}^{\infty} g\left[S_{X}(t)\right] d t=\int_{0}^{u} d t=u
$$

If $u \leq 0$, then

$$
H_{g}(X)=\int_{-\infty}^{0}\left\{g\left[S_{X}(t)\right]-1\right\} d t+\int_{0}^{\infty} g\left[S_{X}(t)\right] d t=\int_{u}^{0}(-1) d t=u
$$

The lemma is proved.

Lemma 2. (Feller, 1971, Chapter 13, Theorem 1a). Let $U$ be a measure on $R^{+}=(0,+\infty)$, taking finite values in bounded sets of $R^{+}$, and

$$
\phi(\lambda)=\int_{0}^{\infty} e^{-\lambda x} U(d x)
$$

be finite for $a<\lambda<\infty$, where $a \geq 0$ is known. Then $U$ is determined by its Laplace transformation $\phi(\lambda)$.

Proposition 1. $\bigcap_{g \in S} N_{g}(X)$ is a location-scale family.

Proof: Let $F=\bigcap_{g \in S} N_{g}(X)$. For any surjective distortion, Wang's premium principle reduces to the $\mathrm{SD}$ premium principle on the set $F$ of distributions. Thus for any $X, Y \in F$ and any surjective distortion $g$, we have

$$
\frac{H_{g}(X)-E(X)}{\sqrt{\operatorname{Var}(X)}}=\frac{H_{g}(Y)-E(Y)}{\sqrt{\operatorname{Var}(Y)}}
$$

Let

$$
U=\frac{X-E(X)}{\sqrt{\operatorname{Var}(X)}}, \quad V=\frac{Y-E(Y)}{\sqrt{\operatorname{Var}(Y)}}
$$

Hence, we have

$$
H_{g}(U)=H_{g}(V)
$$

because $H_{g}$ is location and scale equivariant (or linear invariant). 
Therefore, in order to prove the proposition it is sufficient to show that if the equation (1) holds for all surjective distortions $g, U$ and $V$ have an identical distribution.

Denote the decumulative distribution functions of $U$ and $V$ as $S_{U}(t)$ and $S_{V}(t)$ respectively. Now, using proof by contradiction, we will prove that $S_{U}(t)=S_{V}(t)$ for $t \geq 0$. Assume that there exists a $t_{0} \geq 0$ such that $S_{U}\left(t_{0}\right)>S_{V}\left(t_{0}\right)$. Let $\alpha=S_{V}\left(t_{0}\right), 0 \leq \alpha \leq 1$. Denote $v=\inf \left\{t: S_{V}(t)=\alpha\right\}$, then $v \leq t_{0}$. Take $\epsilon$ such that

$$
0<\epsilon<S_{U}\left(t_{0}\right)-\alpha
$$

and define

$$
\begin{aligned}
& g(w)= \begin{cases}1, & \alpha+\epsilon<w \leq 1 ; \\
(w-\alpha) / \epsilon, & \alpha<w \leq \alpha+\epsilon ; \\
0, & 0 \leq w \leq \alpha .\end{cases} \\
& h_{1}(w)= \begin{cases}1, & \alpha+\epsilon<w \leq 1 ; \\
0, & 0 \leq w \leq \alpha+\epsilon .\end{cases} \\
& h_{2}(w)= \begin{cases}1, & \alpha<w \leq 1 ; \\
0, & 0 \leq w \leq \alpha .\end{cases}
\end{aligned}
$$

Then it is obvious that $g(w)$ is a surjective distortion and $h_{1}(w) \leq g(w) \leq h_{2}(w)$ for all $w \in[0,1]$.

Let $u(\epsilon)=\inf \left\{t: S_{U}(t) \leq \alpha+\epsilon\right\}$, then

$$
S_{U}(u(\epsilon)) \geq \alpha
$$

Since $S_{U}\left(t_{0}\right)>\alpha+\epsilon$, we have

$$
u(\epsilon)>t_{0}
$$

Using Lemma 1, we have

$$
H_{g}(U) \geq H_{h_{1}}(U)=u(\epsilon)
$$

and

$$
H_{g}(V) \leq H_{h_{2}}(V)=v \leq t_{0}<u(\epsilon)
$$

Hence, $H_{g}(U) \neq H_{g}(V)$, contradicting equation (2).

Similar to the proof of proposition 2 in Wang (2000), it can be shown that $S_{U}(t)=S_{V}(t)$ for $t<0$. Hence the proposition is proved.

Proposition 2. If the d.d.f. of $X$ is a continuous function and if the supporting set of $X$ is a convex set, then $\bigcap_{g \in P} N_{g}(X)$ is a location-scale family. 
Proof: Similar to the above proof, in order to prove this proposition it is sufficient to show that if $H_{g}(U)=H_{g}(V)$ for all power distortions $g(p)=p^{c}, U$ and $V$ have an identical distribution where the d.d.f. of both $U$ and $V$ are continuous functions and the supporting sets of both $U$ and $V$ are convex sets.

Let $g$ be a power distortion given by $g(p)=p^{c}$. Now we represent the PH premium $H_{g}(X)$ in the form of a Laplace transformation of random variable $X$ with respect to some function.

$$
\begin{aligned}
H_{g}(X) & =\int_{-\infty}^{0}\left[\left(S_{X}(t)\right)^{c}-1\right] d t+\int_{0}^{\infty}\left(S_{X}(t)\right)^{c} d t \\
& =-\int_{-\infty}^{\infty} t d\left(S_{X}(t)\right)^{c} \\
& =-c \int_{-\infty}^{\infty} t\left(S_{X}(t)\right)^{c-1} d S_{X}(t) \\
& =-c \int_{-\infty}^{\infty} t\left(S_{X}(t)\right)^{c} d\left[\ln S_{X}(t)\right]
\end{aligned}
$$

Let $z=-\ln S_{X}(t)$, then

$$
H_{g}(X)=-c \int_{0}^{\infty} S_{X}^{-1}\left(e^{-z}\right) e^{-c z} d z
$$

which means that $H_{g}(X)$ is the Laplace transformation of $-S_{X}^{-1}\left(e^{-z}\right)$, where $S_{X}^{-1}(\cdot)$ is the inverse function of $S_{X}(\cdot)$. Because the d.d.f. of $X$ is continuous and the supporting set of $X$ is convex, this Laplace transformation exists. Hence, according to Lemma 2, if $H_{g}(U)=H_{g}(V)$, i.e.

$$
\int_{0}^{\infty} S_{U}^{-1}\left(e^{-z}\right) e^{-c z} d z=\int_{0}^{\infty} S_{V}^{-1}\left(e^{-z}\right) e^{-c z} d z
$$

we have

$$
S_{U}^{-1}\left(e^{-z}\right)=S_{V}^{-1}\left(e^{-z}\right)
$$

which implies that $S_{U}(t)=S_{V}(t)$. Hence $U$ and $V$ have an identical distribution. Thus the proof is completed.

To conclude, the PH-transformation principle reduces to the standard deviation principle only on a natural set of location-scale family of distributions. This is merely a result of (and nothing more than) the positive linearty of Wang premium principle. 


\title{
ACKNOWLEDGEMENT
}

The author thanks the anonymous referees for their suggestions that have highly improved the paper.

\section{REFERENCES}

Christofides, S. (1998) Principle for risk in financial transactions. Proceedings of the GISG/ASTIN Joint Meeting in Glasgow, Scotland, October, 1998 2, 63-109.

FELLER, W. (1971) An introduction to probability and its applications. John Wiley \& Sons, Inc. WANG, J.L. (2000) A note on Christofides' conjecture regarding Wang's premium principle. ASTIN Bulletin 30, 13-17.

WANG, S.S. (1995) Insurance pricing and increased limits ratemaking by proportional hazards transforms. Insurance: Mathematics and Economics 17, 43-54.

WANG, S.S. (1996) Premium calculation by transforming the layer premium density. ASTIN Bulletin 26, 71-92.

WANG, S.S. and YounG, V.R. (1998) Ordering risks: Expected utility theory versus Yaari's dual theory of risk. Insurance: Mathematics and Economics 22, 145-161.

WANG, S.S., YoUnG, V.R. and PANJER, H.H. (1997) Axiomatic characterization of insurance price. Insurance: Mathematics and Economics 21, 173-183.

YounG, V.R. (1999) Discussion of Christofides' conjecture regarding Wang's premium principle. ASTIN Bulletin 29, 191-195.

\author{
WU XIAN-YI \\ Department of Statistics \\ East China Normal University \\ 3663 Zhongshan Road (Northern) \\ Shanghai 200062 \\ P.R. China
}

